\title{
Fabrication, Characterization, and Antibacterial Properties of Electrospun Membrane Composed of Gum Karaya, Polyvinyl Alcohol, and Silver Nanoparticles
}

\author{
Vinod Vellora Thekkae Padil, Nhung H. A. Nguyen, Alena Ševců, and Miroslav Černík \\ Institute for Nanomaterials, Advanced Technologies and Innovation, Technical University of Liberec, Studentská 1402/2, Czech Republic
}

Correspondence should be addressed to Miroslav Černík; miroslav.cernik@tul.cz

Received 30 July 2014; Accepted 13 October 2014

Academic Editor: Naeem Faraz

Copyright (c) 2015 Vinod Vellora Thekkae Padil et al. This is an open access article distributed under the Creative Commons Attribution License, which permits unrestricted use, distribution, and reproduction in any medium, provided the original work is properly cited.

\begin{abstract}
Gum karaya (GK), a natural hydrocolloid, was mixed with polyvinyl alcohol (PVA) at different weight ratios and electrospun to produce PVA/GK nanofibers. An $80: 20 \mathrm{PVA} / \mathrm{GK}$ ratio produced the most suitable nanofiber for further testing. Silver nanoparticles (Ag-NPs) were synthesised through chemical reduction of $\mathrm{AgNO}_{3}$ (at different concentrations) in the PVA/GK solution, the GK hydroxyl groups being oxidised to carbonyl groups, and $\mathrm{Ag}^{+}$cations reduced to metallic Ag-NPs. These PVA/GK/Ag solutions were then electrospun to produce nanofiber membranes containing Ag-NPs (Ag-MEMs). Membrane morphology and other characteristics were analysed using scanning electron microscopy coupled with energy dispersive X-ray analysis, transmission electron microscopy, and UV-Vis and ATR-FTIR spectroscopy. The antibacterial activity of the Ag-NP solution and Ag-MEM was then investigated against Gram-negative Escherichia coli and Pseudomonas aeruginosa and Gram-positive Staphylococcus aureus. Our results show that electrospun nanofiber membranes based on natural hydrocolloid, synthetic polymer, and Ag-NPs have many potential uses in medical applications, food packaging, and water treatment.
\end{abstract}

\section{Introduction}

Natural gums derived from plants have many potentially valuable uses as food additives and pharmaceutical ingredients as well as stabilising, suspending, gelling, emulsifying, thickening, binding, and coating agents [1]. In recent years, much research has been undertaken on the application and physicochemical, morphological, and structural properties of exudate gums, such as gum arabic, gum tragacanth, gum karaya, and gum kondagogu [2-5]. Natural biopolymers based on plant exudates have already been used in the preparation of nanoparticles, with gum arabic, for example, having been assessed as a nontoxic phytochemical scaffold for the production of biocompatible gold nanoparticles, which have diagnosis and therapeutic applications [6]. Natural treebased hydrocolloids serve as both an environmentally benign medium and as a chemical reductant, as they have extensive numbers of hydroxyl, carbonyl, and carboxylic groups.
These groups facilitate the formation of metal nanoparticles through the reduction of metal ions and the biopolymer can act as a stabilising agent to prevent nanoparticle agglomeration $[7,8]$. Furthermore, the complex polysaccharide and protein structures of such gums can effectively lock metal nanoparticles to produce nontoxic nanoparticulate products that have a wide range of applications (e.g., in nanomedicine) and are stable under in vivo conditions [9]. Gum karaya (GK), defined by JECFA (Joint Expert Committee for Food Additives) as dried exudates from the stems and branches of Sterculia urens Roxburgh and other species of Sterculia (family: Sterculiaceae), is a partially acetylated polysaccharide with a branched structure and a high molecular mass of $\sim 16 \times 10^{6} \mathrm{Da}$ $[1,10]$. This gum contains about $60 \%$ neutral sugars (rhamnose and galactose) and 40\% acidic sugars (glucuronic and galacturonic acids) [11]. Due to its high viscosity and suspension properties, GK is widely used as a food stabiliser, meat binder, bulk laxative, denture powder, and textile size [1]. 
Electrospinning, an environmentally friendly process capable of producing polymer nanofibers with high porosity and large surface area, allows for the use of a variety of polymers and polymer mixtures together with additives and fillers such as gums $[12,13]$. Nanofibers produced by electrospinning can be further supplemented with a variety of nanoparticles in order to fabricate composites with unique, tailor-made properties for different applications [14]. The "spinnability" and mechanical integrity of natural polymers, such as chitin, chitosan, GK, or ulvan polysaccharide, can be improved by blending with synthetic biodegradable polymers such as polyvinyl alcohol (PVA), polyethylene oxide (PEO), and polyvinylpyrrolidone (PVP) [15-18]. The nature and morphology of the nanofibers produced will be affected by many factors, including the physicochemical properties of the polymer and various parameters of the electrospinning process, including solution viscosity and mixture conductivity [19]. Nanofibers have recently been successfully electrospun using ulvan polysaccharide extracted from an Ulva sp. seaweed blended with PVA [16]. As PVA is a water soluble and biocompatible polymer, it is one of the best materials for preparation of a wide range of potential biomedical materials $[20,21]$.

The properties of silver nanoparticles (Ag-NPs) make them particularly useful as antimicrobial materials, biosensors, composite fibres, cryogenic superconducting materials, cosmetic products, antibacterial medical textiles, wound dressing materials, and electronic components [22-24]. Silver $(\mathrm{Ag})$, especially in nanoparticulate form, is widely recognised as an efficient disinfectant against a wide spectrum of bacteria and viruses and, as such, Ag-NPs (usually between 10 and $20 \mathrm{~nm}$ ) have been used as additives in both natural and synthetic biomedical gels, films, and fibers to improve the antibacterial capability of these materials [25-27]. To date, Ag-NPs have been incorporated into a wide variety of natural or synthetic electrospun nanofibers, including carboxymethyl/chitosan, chitosan/PVA, PVA/gum arabic, PVA/carboxyl methyl/chitosan, PVA/tetraethyl orthosilicate, carboxymethyl chitosan/polyethylene oxide, and curcumin/ chitosan-PVA [28-33]. As an advanced process for generating nanostructures, coaxial electrospinning was also reported to prepare Ag NPs loaded polyacrylonitrile nanofibers [34, 35].

In this study, we describe a method for producing a new nanofiber membrane and film composed of PVA/GK coated with Ag-NPs. We assess the material's morphology using various microscopy and spectroscopy techniques and assess its antibacterial activity using Gram-positive and Gramnegative bacteria. The results are discussed in the light of their potential usefulness in the medical, food packaging, and water treatment industries.

\section{Experimental Section}

2.1. Materials. Commercial gum karaya (partially deacetylated) with molecular weight (Mw: $\left.1.827 \times 10^{6} \mathrm{~g} / \mathrm{mole}\right)$, PVA (Mw 88,000, 88\% deacetylated), silver nitrate $\left(\mathrm{AgNO}_{3}\right)$, and glutaraldehyde solution (Grade 1,50\% in water) were purchased from Sigma-Aldrich, USA. All other reagents used in the experiment were of analytical grade. Deionised water was used throughout.

2.2. Preparation of PVA, GK, and Electrospinning Solutions (PVA/GK). A $10 \mathrm{wt} \%$ aqueous PVA solution and $1 \mathrm{wt} \% \mathrm{GA}$ were prepared in deionised water. A range of PVA/GK electrospinning solutions were produced by mixing PVA (10 wt $\%)$ solution with GK $(1.0 \mathrm{wt} \%)$ at different weight ratios (i.e., $100: 0,90: 10,80: 20,60: 40$, and $50: 50$ ) in order to identify that, giving the best spinnability and most uniform nanofiber size distribution. The mixtures were kept on a magnetic stirrer at $70^{\circ} \mathrm{C}$ for $5 \mathrm{~h}$ to ensure complete dissolution. The solutions were centrifuged to remove any suspended particles prior to electrospinning.

2.3. Preparation of Ag-NP (PVA/GK/Ag Solution). Based on the results of electrospinning different weight ratios of PVA/GK, the most suitable combination was found to be an $80: 20$ weight ratio mix. This was mixed with aqueous $\mathrm{AgNO}_{3}$ solutions of $1,2,4,5$, and $10 \mathrm{mmol} \mathrm{L}^{-1}$ and the resultant solutions stirred at room temperature for $12 \mathrm{~h}$ to obtain homogeneous solutions. Sufficient Ag-NP formation was indicated by a dark yellowish colour, whereupon the PVA/GK/Ag solution was deemed ready for electrospinning and testing for antibacterial activity.

2.4. Preparation of Ag-MEMs. The PVA/GK and PVA/GK/Ag solutionswere electrospun in order to produce nanofiber membranes. All electrospinning was carried out with a Nanospider electrospinning machine (NS IWS500U, Elmarco, Czech Republic) under the following parameters: spinning electrode width $=500 \mathrm{~mm}$, effective nanofiber layer width $=200-500 \mathrm{~mm}$; spinning distance $=130-$ $280 \mathrm{~mm}$, substrate speed $=0.015-1.95 \mathrm{~m} / \mathrm{min}$, process air flow $=20-150 \mathrm{~m}^{3} / \mathrm{h}$, and voltage $0-50 \mathrm{kV}$. The PVA/GK and PVA/GK/Ag (Ag-MEM) membranes were then cross-linked through exposure to glutaraldehyde vapour in desiccators for $12 \mathrm{~h}$. Both the membranes were then heated in an oven for $12 \mathrm{~h}$ at $110^{\circ} \mathrm{C}$ to complete the cross-linking process. Any excess of glutaraldehyde was removed by keeping membranes under vacuum for $24 \mathrm{~h}$.

2.5. Characterization. Formation of Ag-NPs was confirmed through UV-Vis spectroscopy (UV-1601, Shimadzu, Japan) and transmission electron microscopy (TEM; Tecnai F30, Japan; acceleration voltage $15 \mathrm{kV}$ ) was used to analyse Ag-NP size distribution. The morphology of the PVA/GK nanofibers (different weight ratios) and the Ag-MEM was assessed using scanning electron microscopy with energy dispersive $\mathrm{X}$ ray spectroscopy (SEM-EDXA; Zeiss, Ultra/Plus, Germany). Attenuated total reflectance-fourier transform infrared spectroscopy (ATR-FTIR; NICOLET IZ10, Thermo Scientific, USA) was used to characterise the functional groups of PVA, GK, PVA/GK, and Ag-MEM. Conductivity and viscosity of the electrospinning solutions were recorded using a Toledo FG3 electric conductivity meter (Mettler, USA) and a rotational viscometer (Brookfield Engineering Laboratories, 


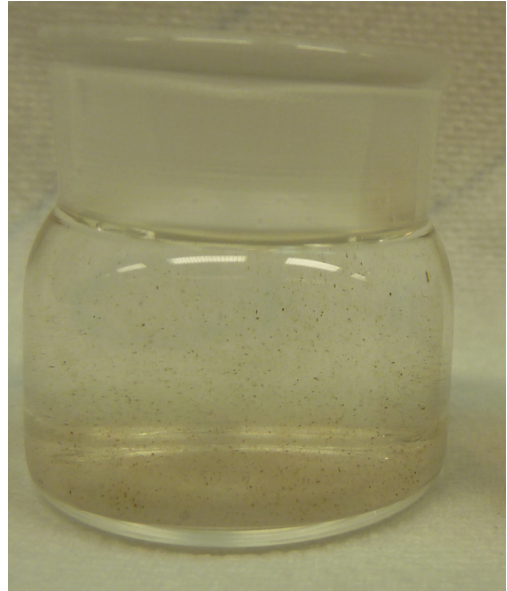

(a)

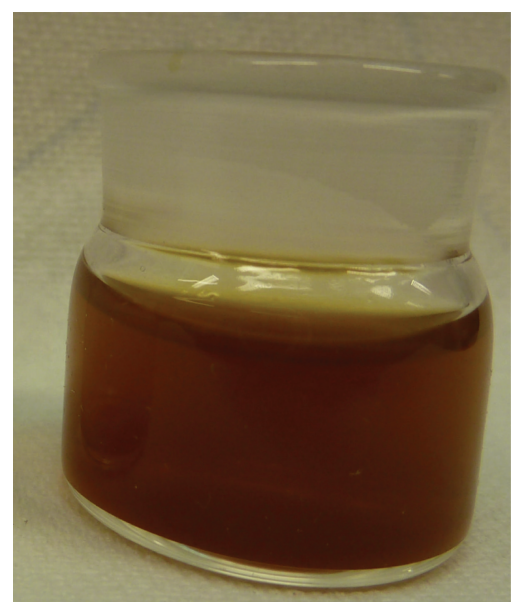

(b)

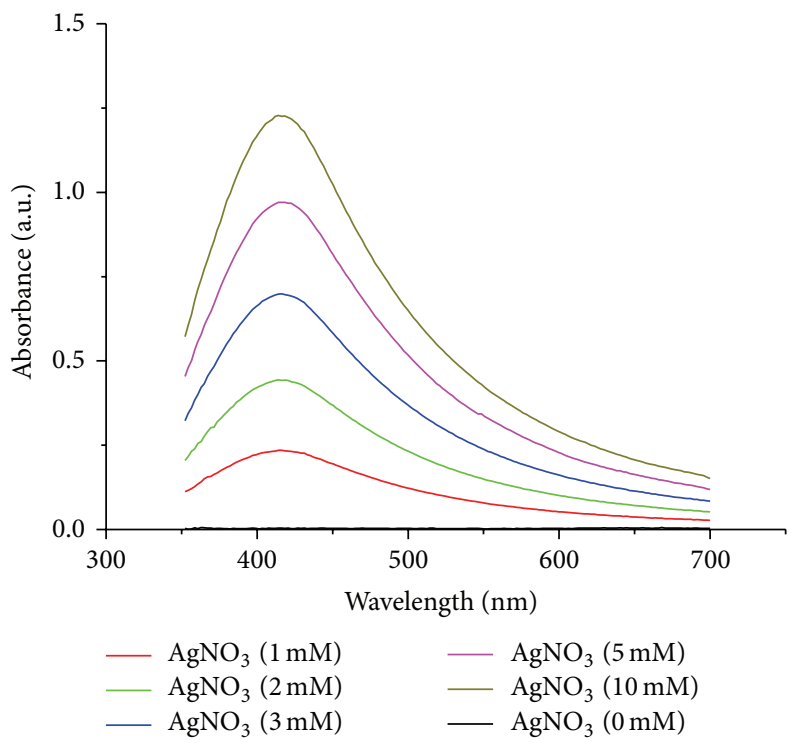

(c)

FIGURE 1: PVA/GK solution (a) before formation of Ag NPs and (b) after Ag NPS formation; and (c) UV-vis spectra of PVA/GK aqueous solution containing Ag-NP prepared at various concentrations of $\operatorname{AgNO}_{3}(0,1,2,4,5$, and $10 \mathrm{mM})$.

USA). The concentration of Ag NPs in PVA/GK/Ag solution and Ag-MEM was established by ICP-AES.

\subsection{Antibacterial Activity Tests}

2.6.1. Bacterial Strains and Culture Media. The bacterial strains of Gram-negative Escherichia coli (CCM 3954) and Pseudomonas aeruginosa (CCM 3955) and Gram-positive Staphylococcus aureus (CCM 3953) used in this study were obtained from the Czech Collection of Microorganisms, Masaryk University Brno, Czech Republic. Bacterial suspensions were always prepared fresh by growing a single colony overnight at $37^{\circ} \mathrm{C}$ in a nutrient broth. The sample turbidity was adjusted to an optical density of 0.1 at 600 (OD 600) before performing the antibacterial experiments. All agar plates were freshly prepared before the antibacterial tests. A sterilised cotton swab was dipped into the culture suspension and the cells spread homogeneously over the agar plates. These plates were immediately used for the antibacterial activity tests.

2.6.2. Determining Zone of Inhibition. We determined the antibacterial activity of four PVA/GK/Ag solutions (1, 2, 4, 5, and $10 \mathrm{mM}$ ) and samples of Ag-MEM (each containing the equivalent of $1 \mathrm{mM}$ of $\mathrm{AgNO}_{3}$ ). The PVA/GK/Ag solutions were pipetted onto a sterilised membrane filter and placed onto an inoculated agar plate, while $6 \mathrm{~mm}$ diameter circles of Ag-MEM were placed directly onto inoculated agar plates. Similarly sized samples of PVA/GK solution $(10 \mathrm{mg} / \mathrm{mL})$ and samples of nanofiber membrane without Ag-NP were used as controls. The samples and inoculated agar plates were then incubated for $24 \mathrm{~h}$ at $37^{\circ} \mathrm{C}$. The zone of inhibition (ZOI) was determined as the total diameter ( $\mathrm{mm}$ ) of PVA/GK/Ag-filter paper or Ag-MEM sample plus the halo zone where bacterial 


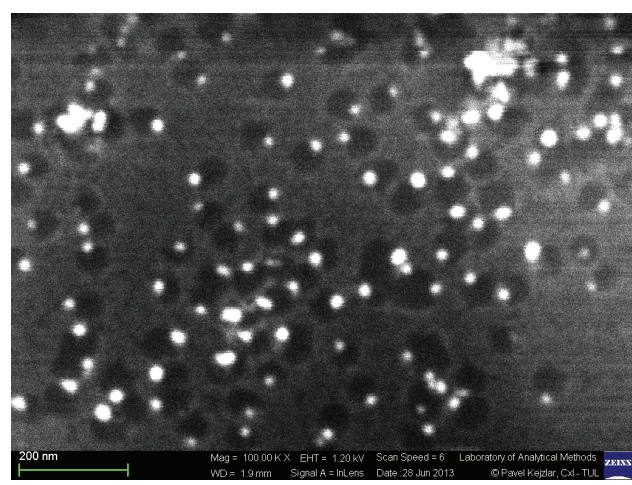

(a)

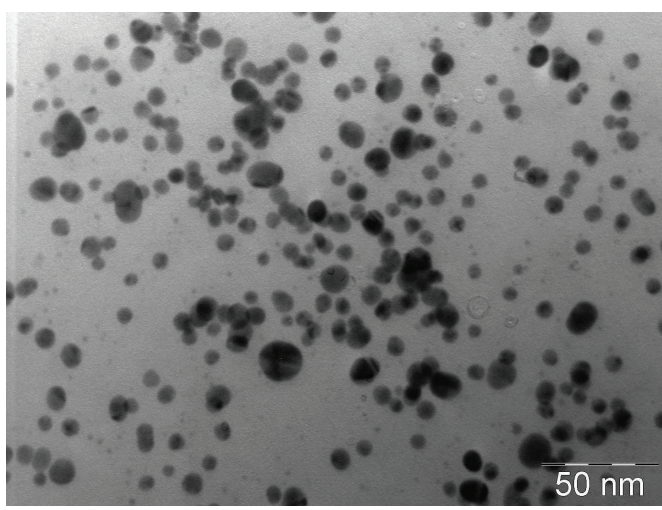

(c)



Map sum spectrum

(b)

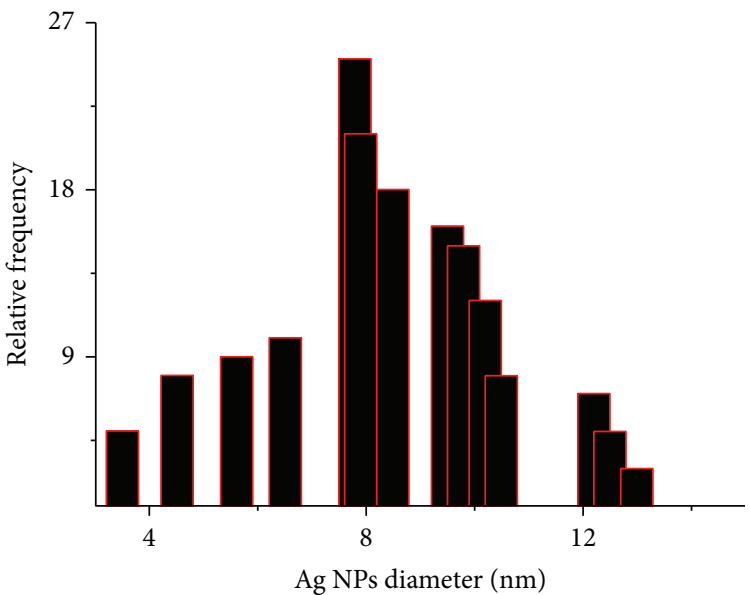

(d)

FIgURE 2: SEM image of (a) Ag-NP prepared using PVA/GK; (b) EDS of Ag-NP, showing the presence of Ag; and (c) TEM image of Ag-NP prepared using PVA/GK and $10 \mathrm{mM} \mathrm{AgNO}_{3}$; and (d) particles diameter distribution of Ag NPs (7-10 nm).

growth was inhibited. All measurements were performed in triplicate for the PVA/GK/Ag solutions and repeated three times (once for each bacterial strain, i.e., nine runs) for the Ag-MEM.

2.7. Statistical Analysis. One-way ANOVA and the MannWhitney test (GraphPad Prism Software, CA, USA) were used to compare differences among the mean ZOIs for the PVA/GK/Ag solutions and Ag-MEM on E. coli, P. aeruginosa, and $S$. aureus.

\section{Results and Discussion}

3.1. Preparation of $A g-N P$ and $P V A / G K$. The colour change of the PVA/GK solution with a ratio of $80: 20$ to dark yellow following formation of Ag-NPs is shown in Figures 1(a) and 1(b). The $420 \mathrm{~nm}$ maximum absorption band seen in the PVA/GK UV-Vis spectra (Figure 1(c)) is a typical plasmon absorption of Ag-NP formation [33].
GK comprises around 60\% neutral sugars and $40 \%$ acidic sugars and a range of hydroxyl, carbonyl, carboxyl, and acetyl functional groups [36]. Following addition of $\mathrm{AgNO}_{3}$, the GK hydroxyl groups are oxidised to carbonyl groups and $\mathrm{Ag}^{+}$ cations are reduced to metallic Ag-NPs. PVA acts as a good stabilising agent for these Ag-NPs due to a free electron pair on the hydroxyl oxygen [31]. Similar observations have been reported for synthesis of Ag-NPs using PVA/carboxymethylchitosan and chitosan/PVA polymer blends and gum arabic/PVA hydrogel $[28,29,31]$.

Presence of Ag-NPs in the PVA/GK/Ag solution was confirmed by SEM imaging of freshly formed AgNP (Figure 2(a)) and its corresponding EDXA analysis (Figure 2(b)). TEM imaging and a particle-size histogram indicate that the majority of the Ag-NPs formed were within a range of 7-10 $\mathrm{nm}$ (Figures 2(c) and 2(d)).

3.2. Electrospinning of $P V A / G K$ and $P V A / G K / A g$. We prepared a range of PVA/GK weight ratio mixtures (100:0, 


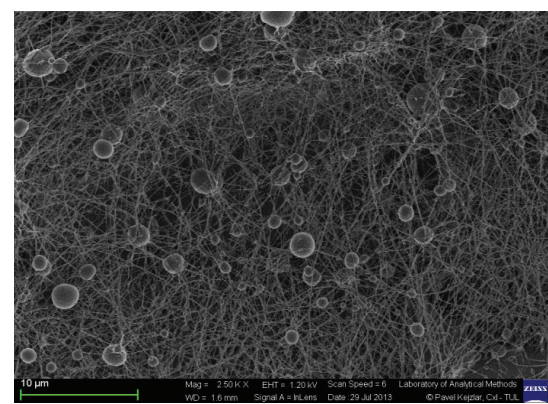

(a)

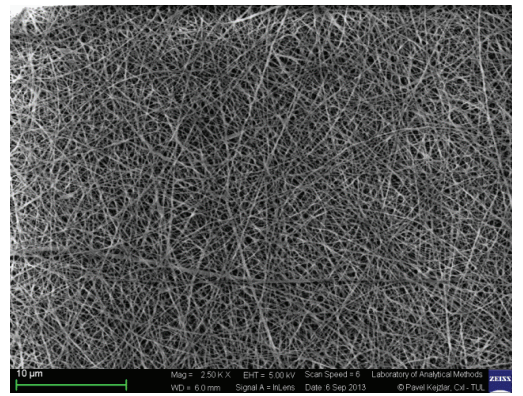

(d)

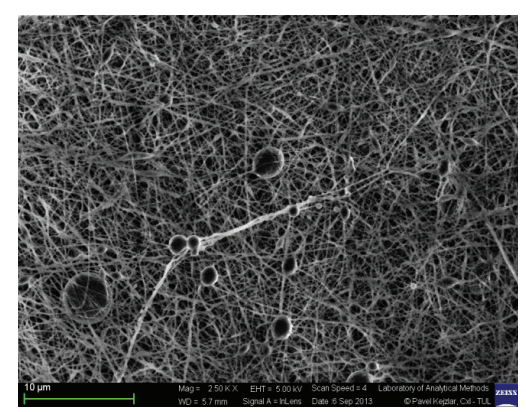

(b)



(e)

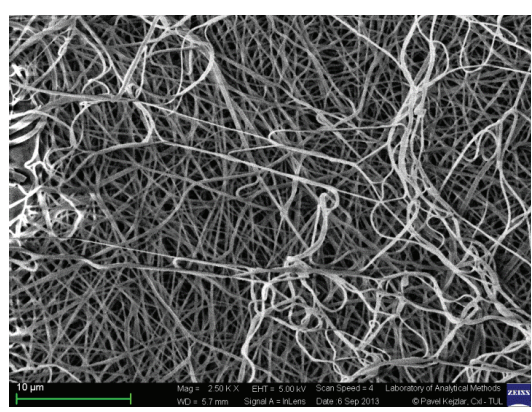

(c)

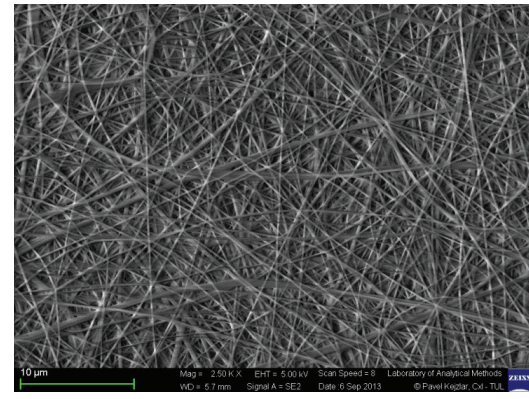

(f)

Figure 3: SEM images of electrospun PVA/GK mixed with different weight ratios: (a) PVA/GK (50/50); (b) PVA/GK (60/40); (c) PVA/GK (70/30); (d) PVA/GK (80/20); (e) PVA/GK (90/10); and (f) neat PVA (100/0) $10 \mu \mathrm{m}$.

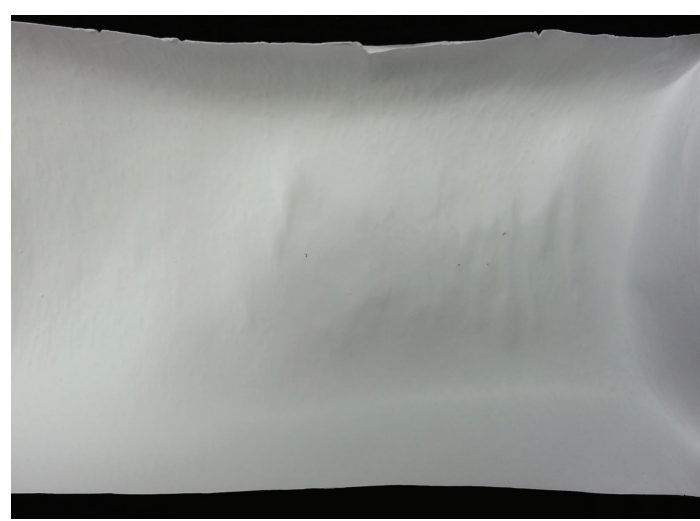

(a)



(b)

FIGURE 4: Digital image of (a) PVA/GK membrane and (b) Ag-MEM prepared by electrospinning of PVA/GK and PVA/GK/Ag NP solution, respectively.

$90: 10,80: 20,60: 40$, and $50: 50)$ in order to optimise the electrospinning solution, that is, to obtain optimal spinnability and uniform nanofiber size. SEM images of the resultant nanofibers (Figures 3(a)-3(f)) indicate that, while nanofibers of pure PVA were uniformly distributed (Figure 3(f)), pure GK fibres could not be electrospun at all due to repulsion from the various highly charged polyanions resulting in chain entanglement.

Further, the pure GK solution proved too viscous for electrospinning as GK is an acidic polymer with high viscosity and molecular weight [8]. Indeed, the PVA/GK blend ratio proved critical in obtaining uniform nanofibers, with evenly formed nanofibers only obtained at PVA/GK weight ratios of $80: 20$ and $90: 10$ (Figures 3(d) and 3(e)). Uniform nanofiber diameters of $200 \mathrm{~nm}$ were only produced at a PVA/GK weight ratio $80: 20$, however; hence, this ratio was selected for all further experiments. Overall, higher PVA/GK ratios enhanced fibre size homogeneity by improving the solubility of the mixed polymers and by decreasing polymer chain aggregation.

Not only were the nature and morphology of the nanofibers affected by polymer solution viscosity and conductivity (both affected by the PVA/GK weight ratio used), but we also found that the viscosity of the PVA/GK/Ag 


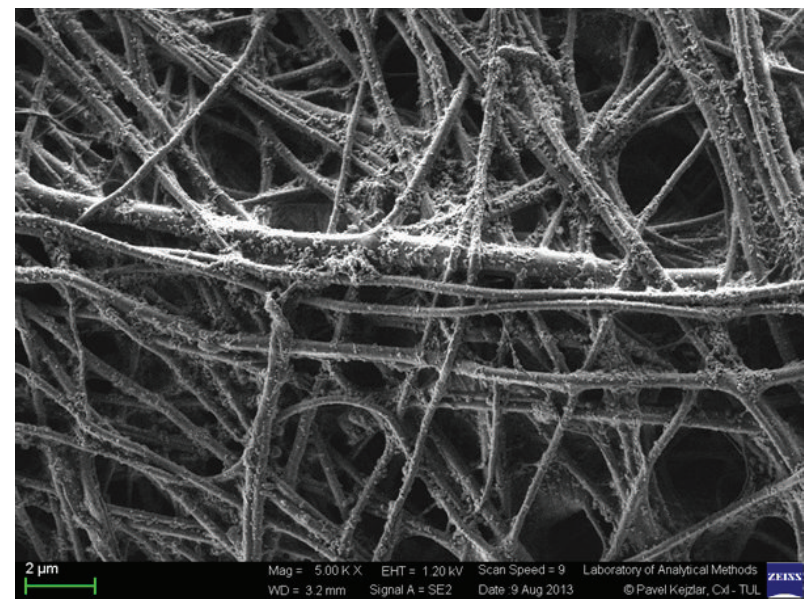

(a)

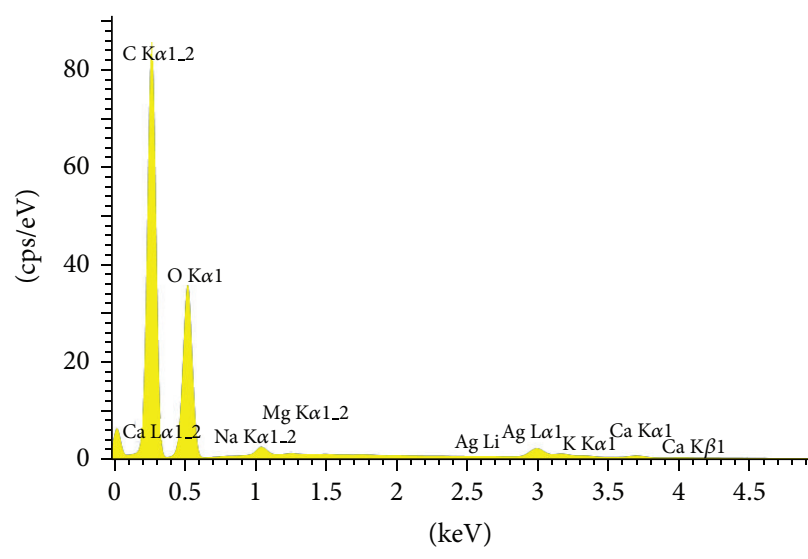

Spectrum 1

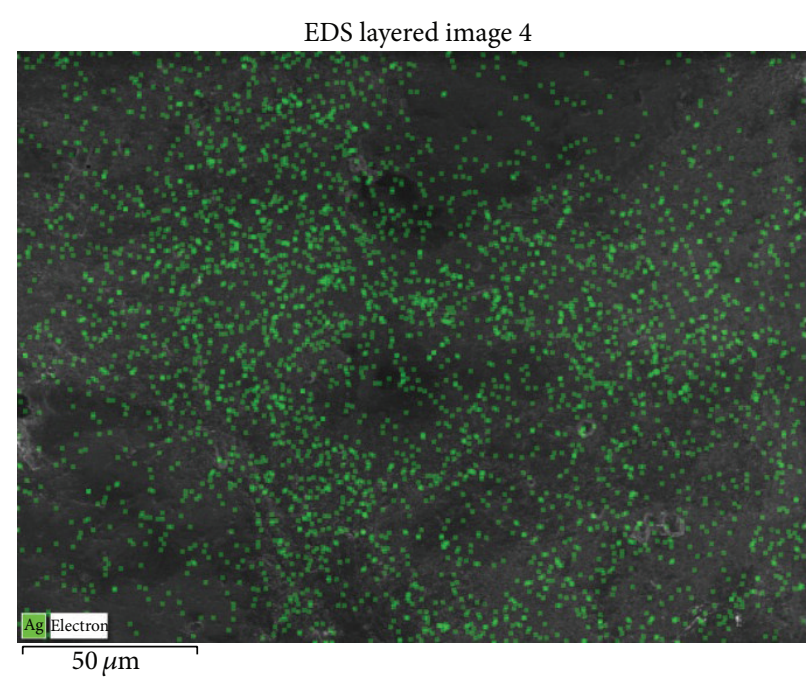

(c)

FIgURE 5: SEM micrograph of (a) Ag-MEM showing the presence of Ag NPs on the surface membrane; (b) EDXA analysis of Ag-NP on Ag-MEM; (c) EDXA layered image indicating the presence of Ag-NP on the surface of Ag-MEM.

electrospinning solution increased from 300 to $500 \mathrm{mPa} \cdot \mathrm{s}$ and its conductivity from 2500 to $3200 \mathrm{mS} \cdot \mathrm{cm}^{-1}$, with increasing $\mathrm{AgNO}_{3}$ concentration $\left(1,2,4,5\right.$, and $\left.10 \mathrm{mmol} \mathrm{L}^{-1}\right)$. The levels at $1 \mathrm{mM}$, however, were within acceptable limits for electrospinning using the $80: 20 \mathrm{PVA} / \mathrm{GK}$ weight ratios and provided reasonable Ag-NP coverage in the final AgMEM (Figure 4(b)) products. The digital photographs of electrospun PVA/GK nanofiber and Ag-MEM are presented in Figures 4(a) and 4(b), respectively.

SEM micrographs of the final electrospun Ag-MEM (Figure 5(a)) clearly show Ag-NPs on the PVA/GK nanofiber surface, and Ag and Ag-NP presence was also confirmed by EDXA analysis (Figure 5(b)) and an EDXA layered image (Figure 5(c)).

3.3. ATR-FTIR Characterisation of Ag-MEM. In examining the bonding between Ag-NPs and the Ag-MEM (also GK, PVA, and PVA/GK) using ATR-FTIR, we noted a broad absorption peak centred around $3318-3350 \mathrm{~cm}^{-1}$ for all samples, attributable to $\mathrm{O}-\mathrm{H}$ stretching vibration in the hydrogen bonded hydroxyl groups (Figure 6).

The peaks at $1430 \mathrm{~cm}^{-1}$ and $1326 \mathrm{~cm}^{-1}$ are characteristic of $\mathrm{O}-\mathrm{H}$ groups and $\mathrm{C}-\mathrm{H}$ deformation vibration in PVA, respectively, while the peak at $1000-1100 \mathrm{~cm}^{-1}$ can be assigned to $\mathrm{C}-\mathrm{O}$ stretching and $\mathrm{O}-\mathrm{H}$ bending vibrations arising from the PVA chain. The appearance of a new peak at $1561 \mathrm{~cm}^{-1}$ in the PVA/GK blend represents $\mathrm{O}-\mathrm{H}$ group deformation vibration with the $\mathrm{H}$ bond, suggesting the formation of an $\mathrm{H}$ bond between PVA and GK when forming the PVA/GK blend. Structurally, GK has abundant hydroxyl groups; hence, $\mathrm{H}$ bonding interactions between GK and PVA occur readily on blending with PVA. The $\mathrm{O}-\mathrm{H}$ bond absorption band at $3300-3500 \mathrm{~cm}^{-1}$ indicates that the $\mathrm{O}-\mathrm{H}$ bond was involved in bonding with the Ag-NPs. Carboxylate group stretching vibration at $1419 \mathrm{~cm}^{-1}$ was considerably reduced in the PVA/GK/Ag-MEM spectrum, demonstrating binding of $\mathrm{Ag}^{+}$ 



Figure 6: ATR-FTIR spectra of PVA, GK, PVA/GK blend, and Ag-MEM.

TABLE 1: Diameter (mm) of zone of inhibition (ZOI) for PVA/GK/Ag solutions produced with different concentrations of $\mathrm{AgNO}_{3}(1,2,4,5$, and $10 \mathrm{mM})$ and $\mathrm{Ag}-\mathrm{MEM}\left(1 \mathrm{mM} \mathrm{AgNO}_{3}\right)$. Means were calculated from in triplicate tests on the PVA/GK/Ag solution and nine replicates for the Ag-MEM $( \pm S D)$.

\begin{tabular}{|c|c|c|c|c|c|c|}
\hline \multirow[b]{2}{*}{$\mathrm{AgNO}_{3}(\mathrm{mM})$} & \multicolumn{5}{|c|}{ PVA/GK/Ag solution } & Ag-MEM \\
\hline & 1 & 2 & 4 & 5 & 10 & 1 \\
\hline E. coli & $8.0 \pm 0.7$ & $9.0 \pm 0.5$ & $10.0 \pm 0.8$ & $11.0 \pm 0.5$ & $14.0 \pm 0.7$ & $7.9 \pm 0.8$ \\
\hline P. aeruginosa & $8.0 \pm 0.8$ & $10.5 \pm 0.8$ & $11.5 \pm 0.7$ & $12.5 \pm 0.7$ & $14.5 \pm 0.7$ & $8.0 \pm 0.7$ \\
\hline S. aureus & $8.1 \pm 0.8$ & $10.5 \pm 0.7$ & $11.5 \pm 0.6$ & $12.5 \pm 0.6$ & $14.5 \pm 0.8$ & $8.0 \pm 0.8$ \\
\hline
\end{tabular}

ions with the PVA/GK nanofibres. These results are in agreement with earlier reported studies on the binding of AgNPs with other natural gums $[7,11]$.

3.4. Antibacterial Properties. We tested the antibacterial activity of the PVA/GK/Ag and Ag-MEM composites synthesised in this study against Gram-negative E. coli and $P$. aeruginosa and Gram-positive $S$. aureus. The results indicate that PVA/GK and Ag-MEM without Ag-NPs show no antibacterial activity.

For the PVA/GK/Ag solution, zone of inhibitions (ZOIs) for Gram-negative $E$. coli and $P$. aeruginosa shows similar antibacterial trends. The ZOI of E. coli increased from 8 to $14 \mathrm{~mm}$ with increasing Ag NPs concentration. Similarly, the ZOI of $P$. aeruginosa increased from 8 to $14.5 \mathrm{~mm}$ with increasing Ag NPs concentration (Table 1).

The ZOI of Gram-positive $S$. aureus increased from 8.5 to $14.5 \mathrm{~mm}$ with increasing Ag NPs concentration (Table 1). Interestingly, growth of both Gram-negative (E. coli and $P$. aeruginosa) and Gram-positive bacteria ( $S$. aureus) was inhibited by the Ag-MEM (ZOI 8), with no significant difference $(P>0.05)$ between the bacterial strains (Table 1 and Figure 7). The concentration of the Ag NPs was observed to be $157.2 \mathrm{mgL}^{-1}$ in both $\mathrm{Ag}$ solution and Ag-MEM (prepared from $1 \mathrm{mM}$ each concentration of $\mathrm{Ag} \mathrm{NO}_{3}$ ) as determined using ICP-AES, respectively. These results were in 




(a)



(c)

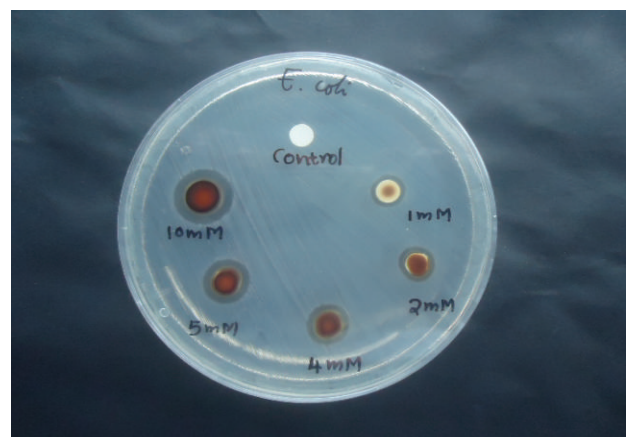

(e)

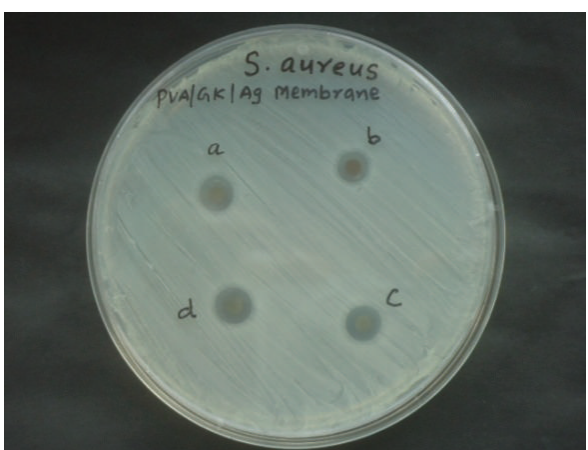

(b)

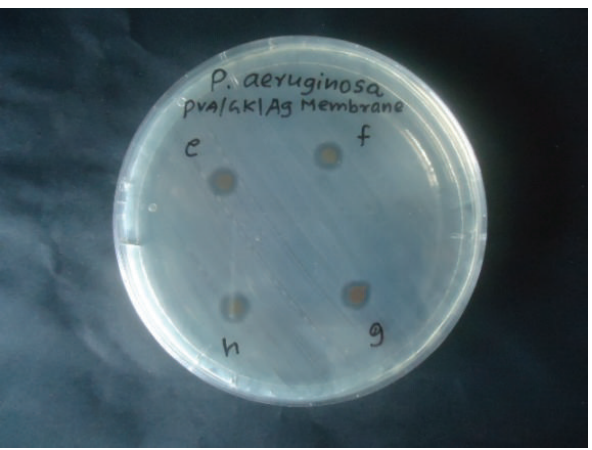

(d)

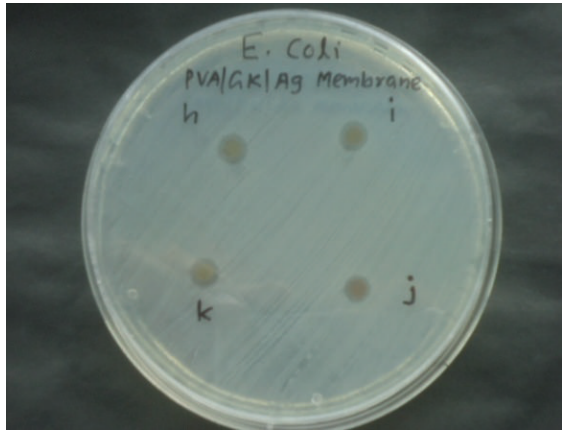

(f)

Figure 7: The bacterial growth inhibition zones of $S$. aureus (a, b), P. aeruginosa (c, d), and E. coli (e, f); left panels represent the antibacterial effect of PVA/GK/Ag NP solution (0, 1, 2, 4, 5 and $10 \mathrm{mM}$ concentration of $\mathrm{AgNO}_{3}$ ) and right panels of Ag-MEM (quadruplicates represent the zone of inhibition of $1 \mathrm{mM}$ concentration of $\mathrm{AgNO}_{3}$ ), respectively.

a good agreement with earlier reported investigation onto antibacterial properties of PVA/Ag NPS/TEOS films and PVA/carboxymethyl-chitosan/AgNanofibers [27, 31].

While the mechanism for Ag-NP action is still not fully understood, it has been documented that Ag-NPs cause structural changes when they interact with the outer membrane of bacteria [37]. Such changes may lead to an increase in membrane permeability and leakage of intracellular constituents and cause severe damage, ultimately resulting in cell death. Differences in bacterial susceptibility may be due to structural and compositional differences in the cell membrane of Gram-positive and Gram-negative bacteria [38, 39]. Gram-negative E. coli cell walls, for example, have dynamic lipopolysaccharide $\mathrm{O}$-side chains that are not present in Gram-positive cell walls. Rapidly moving side chains may disable the formation of a metal-ion salt bridge and prevent an antibiotic effect when Ag-NPs are not present in sufficient concentration [40]. The present investigation showed that Ag-MEM and Ag solution with Ag NP concentration $\left(157.2 \mathrm{mgL}^{-1}\right)$ indicate almost similar zone of inhibition $(\sim 8)$ against both Gram-negative (E. coli and P. aeruginosa) and Gram-positive bacteria ( $S$. aureus). The PVK/GK/Ag solution and Ag-MEM show high potential as environmentally friendly antibacterial materials for a variety of applications, such as medical wound dressings and cosmetics.

\section{Conclusions}

In this study, we produced an electrospun nanofiber membrane from GK, a natural hydrocolloid, blended with PVA. 
Uniform PVA/GK nanofibers were obtained at a PVA/GK weight ratio of $80: 20$. The $80: 20 \mathrm{PVA} / \mathrm{GK}$ was blended with various concentrations of $\mathrm{AgNO}_{3}$ solution to produce a PVA/GK/Ag NP solution. PVA/GK/Ag NP solution was then used to produce nanofibers containing AG-NPs, from which an antibacterial nanofiber membrane (Ag-MEM) was fabricated. The PVA/GK/Ag solution and Ag-MEM showed clear antibacterial activity toward Gram-negative E. coli and P. aeruginosa and Gram-positive S. aureus. As all bacterial species showed similar susceptibility to AgMEM, they show bactericidal action toward a wide range of potentially pathogenic bacteria. These newly synthesised Ag NP solutions and Ag-MEM show great potential for the development of environmentally friendly antibacterial materials for medical devices, food packaging, and water purification purposes.

\section{Conflict of Interests}

The authors declare no conflict of interests.

\section{Acknowledgments}

The research reported in this paper was supported in part by Project LO1201, the financial support of the Ministry of Education, Youth and Sports in the framework of the targeted support of the "National Programme for Sustainability I," the OPR \& DI Project and OP VaVpI of the Centre for Nanomaterials, Advanced Technologies and Innovation, CZ.1.05/2.1.00/01.0005, the "Project Development of Research Teams for R \& D Projects" at the Technical university of Liberec, CZ.1.07/2.3.00/30.0024, and a Grant from the Competence Centre, TE01020218.

\section{References}

[1] D. Verbeken, S. Dierckx, and K. Dewettinck, "Exudate gums: occurrence, production, and applications," Applied Microbiology and Biotechnology, vol. 63, no. 1, pp. 10-21, 2003.

[2] D. M. W. Anderson and W. Weiping, "Gum arabic (Acacia senegal) from Uganda: characterization, NMR spectra, amino acid composite and gum/soil cationic relationship," International Tree Crops Journal, vol. 7, pp. 167-179, 1992.

[3] G. O. Aspinall, L. Khondo, and B. A. Williams, "The hex5-enose degradation: cleavage of glycosiduronic acid linkages in modified methylated Sterculia gums," Canadian Journal of Chemistry, vol. 65, no. 9, pp. 2069-2076, 1986.

[4] M. A. Mohammadifar, S. M. Musavi, A. Kiumarsi, and P. A. Williams, "Solution properties of targacanthin (water-soluble part of gum tragacanth exudate from Astragalus gossypinus)," International Journal of Biological Macromolecules, vol. 38, no. 1, pp. 31-39, 2006.

[5] V. T. P. Vinod, R. B. Sashidhar, K. I. Suresh, B. R. Rao, U. V. R. V. Saradhi, and T. O. Rao, "Morphological, physico-chemical and structural characterization of gum kondagogu (Cochlospermum gossypium): a tree gum from India," Food Hydrocolloids, vol. 22, no. 5, pp. 899-915, 2008.

[6] V. Kattumuri, K. Katti, S. Bhaskaran et al., "Gum arabic as a phytochemical construct for the stabilization of gold nanoparticles: in vivo pharmacokinetics and X-ray-contrast-imaging studies," Small, vol. 3, no. 2, pp. 333-341, 2007.

[7] V. T. P. Vinod, P. Saravanan, B. Sreedhar, D. K. Devi, and R. B. Sashidhar, "A facile synthesis and characterization of Ag, $\mathrm{Au}$ and $\mathrm{Pt}$ nanoparticles using a natural hydrocolloid gum kondagogu (Cochlospermum gossypium)," Colloids and Surfaces B: Biointerfaces, vol. 83, no. 2, pp. 291-298, 2011.

[8] V. V. T. Padil and M. Černík, "Green synthesis of copper oxide nanoparticles using gum karaya as a biotemplate and their antibacterial application," International Journal of Nanomedicine, vol. 8, pp. 889-898, 2013.

[9] R. Arvizo, R. Bhattacharya, and P. Mukherjee, "Gold nanoparticles: opportunities and challenges in nanomedicine," Expert Opinion on Drug Delivery, vol. 7, no. 6, pp. 753-763, 2010.

[10] A. M. Stephen and C. S. C. A. M. Stephen, Eds., Gums and Mucilages, Marcel Dekker, New York, NY, USA, 1995.

[11] D. A. Silva, A. C. F. Brito, R. C. M. de Paula, J. P. A. Feitosa, and H. C. B. Paula, "Effect of mono and divalent salts on gelation of native, $\mathrm{Na}$ and deacetylated Sterculia striata and Sterculia urens polysaccharide gels," Carbohydrate Polymers, vol. 54, no. 2, pp. 229-236, 2003.

[12] T. J. Sill and H. A. von Recum, "Electrospinning: applications in drug delivery and tissue engineering," Biomaterials, vol. 29, no. 13, pp. 1989-2006, 2008.

[13] P. Schexnailder and G. Schmidt, "Nanocomposite polymer hydrogels," Colloid and Polymer Science, vol. 287, no. 1, pp. 1-11, 2009.

[14] V. Thavasi, G. Singh, and S. Ramakrishna, "Electrospun nanofibers in energy and environmental applications," Energy and Environmental Science, vol. 1, no. 2, pp. 205-221, 2008.

[15] M. Z. Elsabee, H. F. Naguib, and R. E. Morsi, "Chitosan based nanofibers, a review," Materials Science and Engineering $C$, vol. 32, no. 7, pp. 1711-1726, 2012.

[16] G. Toskas, R.-D. Hund, E. Laourine, C. Cherif, V. Smyrniotopoulos, and V. Roussis, "Nanofibers based on polysaccharides from the green seaweed Ulva Rigida," Carbohydrate Polymers, vol. 84, no. 3, pp. 1093-1102, 2011.

[17] M. Ignatova, K. Starbova, N. Markova, N. Manolova, and I. Rashkov, "Electrospun nano-fibre mats with antibacterial properties from quaternised chitosan and poly(vinyl alcohol)," Carbohydrate Research, vol. 341, no. 12, pp. 2098-2107, 2006.

[18] M. Pakravan, M. C. Heuzey, and A. Ajji, "A fundamental study of chitosan/PEO electrospinning," Polymer, vol. 52, no. 21, pp. 4813-4824, 2011.

[19] C. Zhang, X. Yuan, L. Wu, Y. Han, and J. Sheng, "Study on morphology of electrospun poly(vinyl alcohol) mats," European Polymer Journal, vol. 41, no. 3, pp. 423-432, 2005.

[20] E. Yang, X. Qin, and S. Wang, "Electrospun crosslinked polyvinyl alcohol membrane," Materials Letters, vol. 62, no. 20, pp. 3555-3557, 2008.

[21] A. Ravindran, P. Chandran, and S. S. Khan, "Bio-functionalized silver nanoparticles: advances and prospects," Colloids and Surfaces B: Biointerfaces, vol. 105, pp. 342-352, 2013.

[22] O. D. Choi, "The inhibitory effects of silver nanoparticles, silver ions, and silver chloride colloids on microbial growth," Water Research, vol. 42, no. 12, pp. 3066-3074, 2008.

[23] S. Porel, D. Ramakrishna, E. Hariprasad, A. D. Gupta, and T. P. Radhakrishnan, "Polymer thin film with in situ synthesized silver nanoparticles as a potent reusable bactericide," Current Science, vol. 101, no. 7, pp. 927-934, 2011. 
[24] P.-O. Rujitanaroj, N. Pimpha, and P. Supaphol, "Wounddressing materials with antibacterial activity from electrospun gelatin fiber mats containing silver nanoparticles," Polymer, vol. 49, no. 21, pp. 4723-4732, 2008.

[25] X. Zhuang, B. Cheng, W. Kang, and X. Xu, "Electrospun chitosan/gelatin nanofibers containing silver nanoparticles," Carbohydrate Polymers, vol. 82, no. 2, pp. 524-527, 2010.

[26] M. S. Peresin, Y. Habibi, J. O. Zoppe, J. J. Pawlak, and O. J. Rojas, "Nanofiber composites of polyvinyl alcohol and cellulose nanocrystals: manufacture and characterization," Biomacromolecules, vol. 11, no. 3, pp. 674-681, 2010.

[27] R. Bryaskova, D. Pencheva, G. M. Kale, U. Lad, and T. Kantardjiev, "Synthesis, characterisation and antibacterial activity of PVA/TEOS/Ag-Np hybrid thin films," Journal of Colloid and Interface Science, vol. 349, no. 1, pp. 77-85, 2010.

[28] K. A. Juby, C. Dwivedi, M. Kumar, S. Kota, H. S. Misra, and P. N. Bajaj, "Silver nanoparticle-loaded PVA/gum acacia hydrogel: synthesis, characterization and antibacterial study," Carbohydrate Polymers, vol. 89, no. 3, pp. 906-913, 2012.

[29] C. W. Li, R. Q. Fu, C. P. Yu et al., "Silver nanoparticle/chitosan oligosaccharide/poly(vinyl alcohol) nanofibers as wound dressings: a preclinical study," International Journal of Nanomedicine, vol. 8, pp. 4131-4145, 2013.

[30] C. Marambio-Jones and E. M. V. Hoek, "A review of the antibacterial effects of silver nanomaterials and potential implications for human health and the environment," Journal of Nanoparticle Research, vol. 12, no. 5, pp. 1531-1551, 2010.

[31] Y. Zhao, Y. Zhou, X. Wu, L. Wang, L. Xu, and S. Wei, "A facile method for electrospinning of Ag nanoparticles/poly (vinyl alcohol)/carboxymethyl-chitosan nanofibers," Applied Surface Science, vol. 258, no. 22, pp. 8867-8873, 2012.

[32] K. Vimala, Y. M. Mohan, K. Varaprasad et al., "Fabrication of curcumin encapsulated chitosan-PVA silver nanocomposite films for improved antimicrobial activity," Journal of Biomaterials and Nanobiotechnology, vol. 2, pp. 55-64, 2011.

[33] H. H. Huang, X. P. Ni, G. L. Loy et al., "Photochemical formation of silver nanoparticles in poly(N-vinylpyrrolidone)," Langmuir, vol. 12, no. 4, pp. 909-912, 1996.

[34] D.-G. Yu, G. R. Williams, X. Wang, X.-K. Liu, H.-L. Li, and S. W. A. Bligh, "Dual drug release nanocomposites prepared using a combination of electrospraying and electrospinning," RSC Advances, vol. 3, no. 14, pp. 4652-4658, 2013.

[35] D.-G. Yu, J. Zhou, N. P. Chatterton, Y. Li, J. Huang, and X. Wang, "Polyacrylonitrile nanofibers coated with silver nanoparticles using a modified coaxial electrospinning process," International Journal of Nanomedicine, vol. 7, pp. 5725-5732, 2012.

[36] V. T. P. Vinod, R. B. Sashidhar, V. U. M. Sarma, and S. S. Raju, "Comparative amino acid and fatty acid compositions of edible gums kondagogu (Cochlospermum gossypium) and karaya (Sterculia urens)," Food Chemistry, vol. 123, no. 1, pp. 5762, 2010.

[37] I. Sondi and B. Salopek-Sondi, "Silver nanoparticles as antimicrobial agent: a case study on E. coli as a model for Gramnegative bacteria," Journal of Colloid and Interface Science, vol. 275, no. 1, pp. 177-182, 2004.

[38] T. J. Beveridge, "Structures of gram-negative cell walls and their derived membrane vesicles," Journal of Bacteriology, vol. 181, no. 16, pp. 4725-4733, 1999.

[39] V. Vadillo-Rodriguez, S. R. Schooling, and J. R. Dutcher, "In situ characterization of differences in the viscoelastic response of individual gram-negative and gram-positive bacterial cells," Journal of Bacteriology, vol. 191, no. 17, pp. 5518-5525, 2009.
[40] S. Langley and T. J. Beveridge, "Effect of O-side-chainlipopolysaccharide chemistry on metal binding," Applied and Environmental Microbiology, vol. 65, no. 2, pp. 489-498, 1999. 

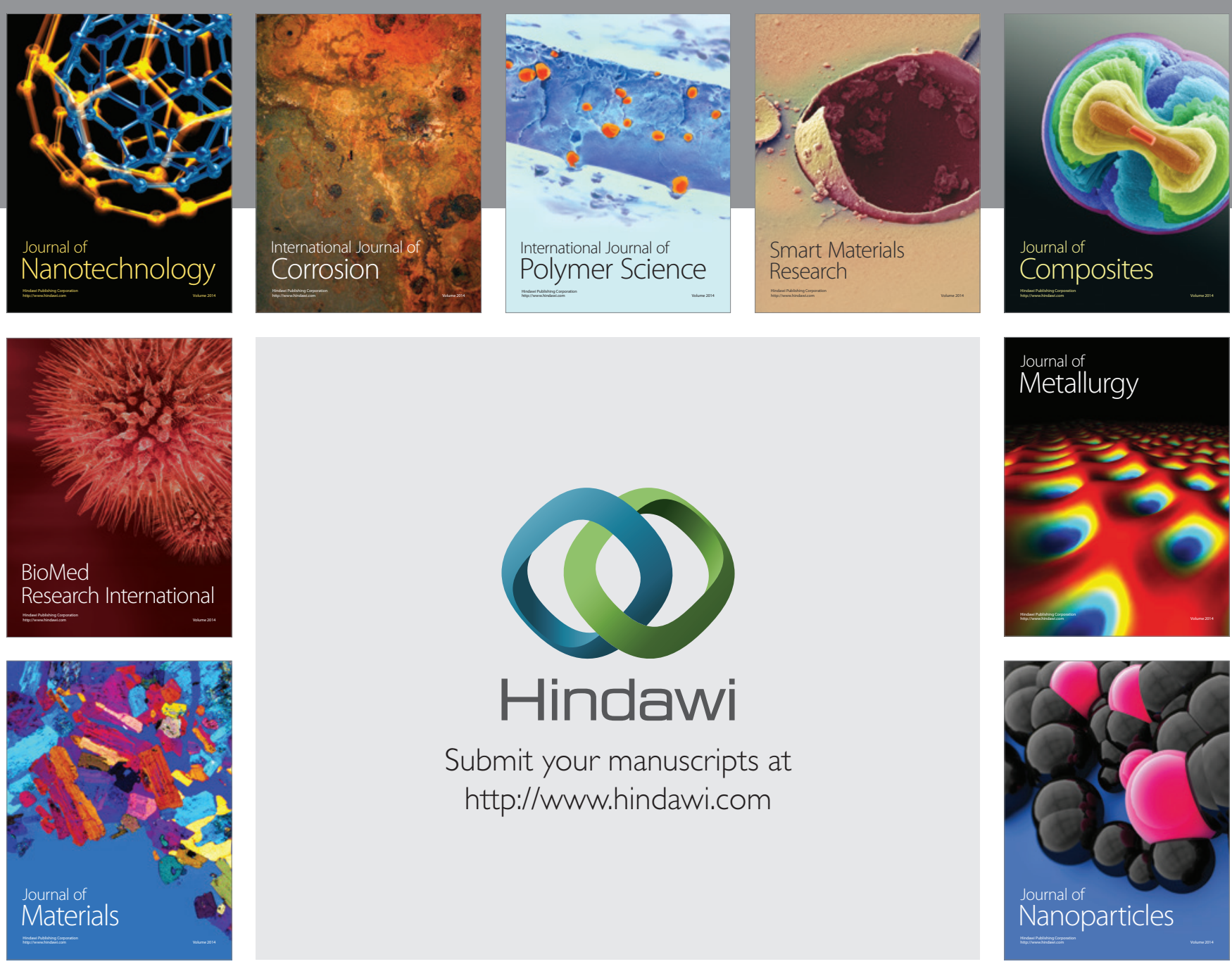

Submit your manuscripts at http://www.hindawi.com


\section{The Scientific World Journal}
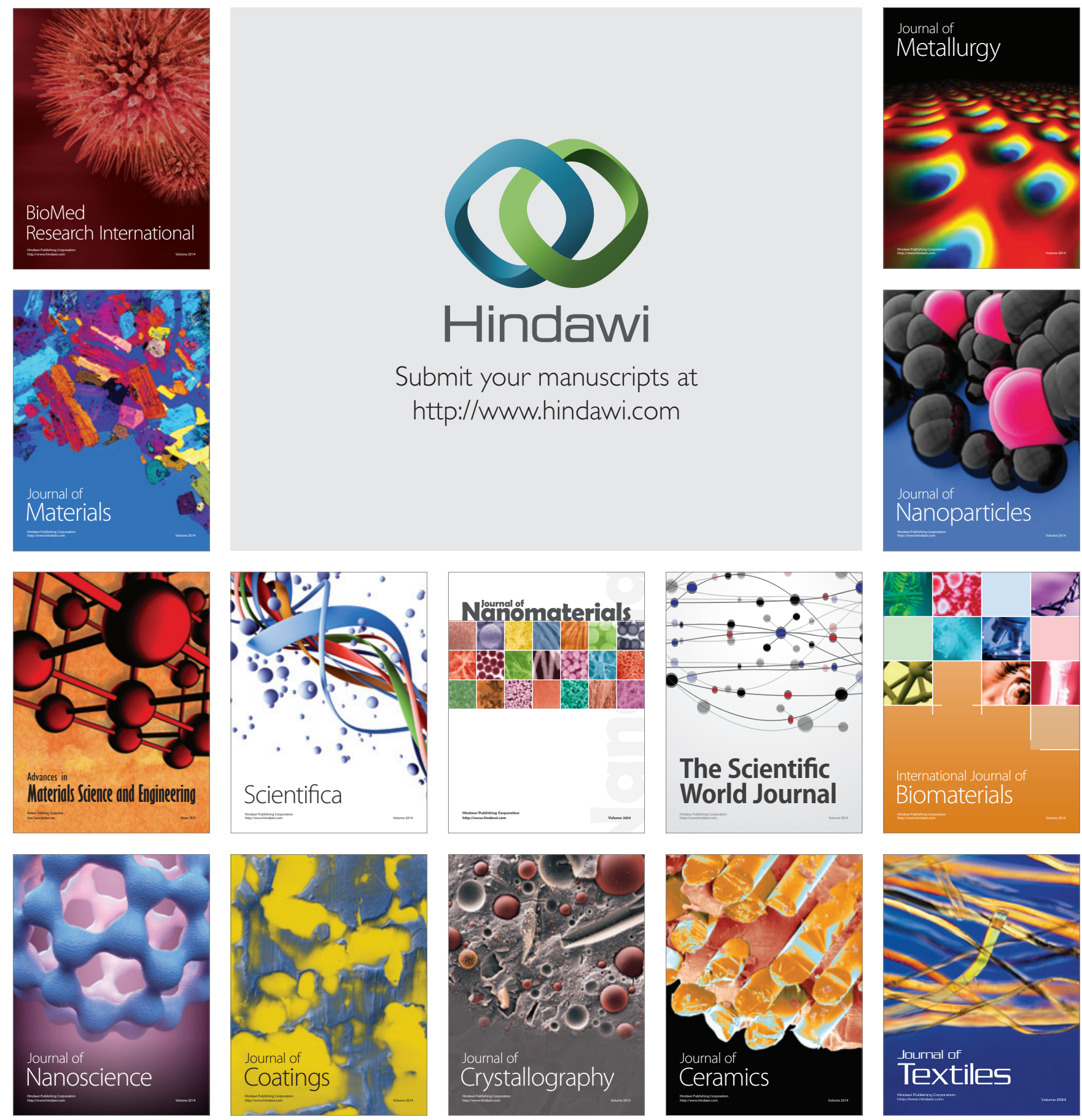